\title{
Correction to: On the topological mass lattice groups
}

\author{
M. Pourgholamhossein ${ }^{1}$ - M. A. Ranjbar ${ }^{1}$
}

Published online: 8 May 2020

(c) Springer Nature Switzerland AG 2020

Keywords Lattice group · Mass group

\section{Correction to: Positivity (2019) 23:811-827 https://doi.org/10.1007/s11117-018-0639-5}

(i) In the first assertion of the Lemma 1 in [2], it is necessary that $e \leq y$. Note. By adding the above condition in the first assertion of the Lemma 1, this assertion holds in every unperforated partially ordered abelian group. In fact the condition lattice is redundant (see [1], p. 19 and Proposition 1.24).

(ii) In the middle of page 819 of [2] (after the definition 9), the assertion "It is clear that $G$ is Archimedean, if and only if, every element of $G$ is regular" is not true without condition mass on $G$. In fact the corrected statement is as follows.

- One can see that if $G$ is Archimedean, then every element of $G$ is regular and the converse is not true in general. But, if $G$ is a mass group, then the converse is true.

\section{References}

1. Goodearl, K.R.: Partially Ordered Abelian Groups with Interpolation, A.M.S. Mathematical Surveys and Monographs, No. 20, American Mathematical Society, Providence, RI, 1986. MR 0845783 (88f:06013)

2. Pourgholamhossein, M., Ranjbar, M.A.: On the topological mass lattice groups. Positivity 23(4), 811827 (2019)

The original article can be found online at https://doi.org/10.1007/s11117-018-0639-5.

M. Pourgholamhossein m-purghol@qom.ac.ir

M. A. Ranjbar ma.Ranjbar@stu.qom.ac.ir

1 Department of Mathematics, Faculty of Sciences, University of Qom, Qom, Iran 
Publisher's Note Springer Nature remains neutral with regard to jurisdictional claims in published maps and institutional affiliations. 